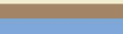

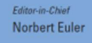

Journal of Nonlinear Mathematical Physics

ISSN (Online): 1776-0852 ISSN (Print): 1402-9251

Journal Home Page: https://www.atlantis-press.com/journals/jnmp

\title{
A multi-symplectic numerical integrator for the two-component Camassa-Holm equation
}

David Cohen, Takayasu Matsuo, Xavier Raynaud

To cite this article: David Cohen, Takayasu Matsuo, Xavier Raynaud (2014) A multisymplectic numerical integrator for the two-component Camassa-Holm equation, Journal of Nonlinear Mathematical Physics 21:3, 442-453, DOI:

https://doi.org/10.1080/14029251.2014.936763

To link to this article: https://doi.org/10.1080/14029251.2014.936763

Published online: 04 January 2021 


\title{
A multi-symplectic numerical integrator for the two-component Camassa-Holm equation
}

\author{
David Cohen \\ Matematik och matematisk statistik, Umeå universitet \\ 90187 Umeå, Sweden \\ david.cohen@math.umu.se \\ Takayasu Matsuo \\ Department of Mathematical Informatics, Graduate School of System of Information Science and Technology, \\ The University of Tokyo, 7-3-1 Hongo Bunkyo-ku \\ 113-8656 Tokyo, Japan \\ matsuo@mist.i.u-tokyo.ac.jp \\ Xavier Raynaud \\ Applied Mathematics, SINTEF ICT \\ Forskningsveien 1, \\ 0373 Oslo, Norway \\ and \\ Department of Mathematical Science, NTNU \\ 7491 Trondheim, Norway \\ xav.raynaud@gmail.com
}

Received 19 February 2014

Accepted 16 May 2014

\begin{abstract}
A new multi-symplectic formulation of the two-component Camassa-Holm equation $(2 \mathrm{CH})$ is presented, and the associated local conservation laws are shown to correspond to certain well-known Hamiltonian functionals. A multi-symplectic discretisation based on this new formulation is exemplified by means of the Euler box scheme. Furthermore, this scheme preserves exactly two discrete versions of the Casimir functions of $2 \mathrm{CH}$. Numerical experiments show that the proposed numerical scheme has good conservation properties.
\end{abstract}

Keywords: Two-component Camassa-Holm equation; Hamiltonian PDE; Casimir function; Numerical discretisation; Multi-symplectic formulation; Multi-symplectic schemes; Euler box scheme.

2010 Mathematics Subject Classification: 35Q51, 35Q53, 37K05, 37K10, 37M15, 65M06, 65M99, 65P10

\section{Introduction}

The goal of this note is to present a multi-symplectic formulation of the two-component CamassaHolm equation (or $2 \mathrm{CH}$ system)

$$
\begin{aligned}
& u_{t}-u_{t x x}+3 u u_{x}=2 u_{x} u_{x x}+u u_{x x x}-\kappa \rho \rho_{x}, \\
& \rho_{t}+(u \rho)_{x}=0,
\end{aligned}
$$

which will allow us to numerically discretise this partial differential equation with a multisymplectic integrator based on the Euler box scheme. In the above equations, we use the notations $u=u(x, t), \rho=\rho(x, t)$ and $\kappa$ denotes a real parameter. The case $\kappa>0$ is the physical relevant 
case. We consider initial conditions $(u(x, 0), \rho(x, 0))=\left(u_{0}, \rho_{0}\right)$ and periodic boundary conditions $u(0, t)=u(L, t)$ and $\rho(0, t)=\rho(L, t)$ on a domain $[0, L]$, for an $L \in \mathbb{R}_{+}$.

The $2 \mathrm{CH}$ system is a generalisation of the famous Camassa-Holm equation (taking $\rho_{0}=0$ as initial value for (1.1)). This latter equation, which possesses a lot of interesting properties, has been extensively studied in the literature. One may consult for example the work [27], the recent review [16], and references therein.

Since its introduction in the seminal paper [26] (eq. (43)), the $2 \mathrm{CH}$ system (1.1) has also received considerable attention. The two-component Camassa-Holm equation approximates the governing equations for shallow water waves [7]. Furthermore, this system of partial differential equations has a rich mathematical structure: it is integrable; has a Lax pair; is bi-Hamiltonian with Hamiltonian functions

$$
\begin{aligned}
& \mathscr{H}_{1}=\frac{1}{2} \int\left(u^{2}+u_{x}^{2}+\kappa \rho^{2}\right) \mathrm{d} x, \\
& \mathscr{H}_{2}=\frac{1}{2} \int\left(u^{3}+u u_{x}^{2}+\kappa u \rho^{2}\right) \mathrm{d} x
\end{aligned}
$$

and with the respective compatible Poisson brackets

$$
\begin{aligned}
& \{F, G\}_{1}=-\int\left(\frac{\delta F}{\delta m}(m \partial+\partial m) \frac{\delta G}{\delta m}+\frac{\delta F}{\delta m} \rho \partial \frac{\delta G}{\delta \rho}+\frac{\delta F}{\delta \rho} \partial \rho \frac{\delta G}{\delta m}\right) \mathrm{d} x, \\
& \{F, G\}_{2}=-\int\left(\frac{\delta F}{\delta m}\left(\partial-\partial^{3}\right) \frac{\delta G}{\delta m}+\frac{\delta F}{\delta \rho} \partial \frac{\delta G}{\delta \rho}\right) \mathrm{d} x,
\end{aligned}
$$

where $F$ and $G$ are two functionals of the variables $m:=u-u_{x x}$ and $\rho$; possesses the following two Casimir functions (for the second Poisson bracket $\{\cdot, \cdot\}_{2}$ )

$$
\begin{aligned}
& \mathscr{C}_{1}=\int \rho \mathrm{d} x, \\
& \mathscr{C}_{2}=\int\left(u-u_{x x}\right) \mathrm{d} x ;
\end{aligned}
$$

has global solutions for small initial data but wave breaking may also occur; admits solitary wave solutions; it can be seen as geodesic equations on some space; this system also appears in plasma theory models and in the theory of metamorphosis; etc. [3, 7, 8, 13-15, 17, 18, 21,24] (without being exhaustive).

The main objective of this note is to present an additional property of the two-component Camassa-Holm equation, namely the multi-symplectic structure of this partial differential equation, see Section 2. This new structure will then be used to derive a multi-symplectic numerical method (the Euler box scheme) in Section 3 in order to approximate solutions of the $2 \mathrm{CH}$ system. Furthermore, we will show that our scheme preserves exactly two discrete versions of the above Casimir functions. Numerical experiments are presented in Section 4 and finally, discussions and conclusions are drawn.

Our derivations of a multi-symplectic formulation of (1.1) and of a multi-symplectic scheme for the $2 \mathrm{CH}$ system follow the lines of [6]. We obtain the same formulation, resp. numerical scheme, as the one presented in [6] when $\rho \equiv 0$ in (1.1). However, since we are not aware of any numerical schemes for the two-component Camassa-Holm equation, except the numerical simulations presented in [15], we think that the results presented in this short note are of interest and hope that they will initiate further numerical analysis of the $2 \mathrm{CH}$ system. 


\section{Multi-symplectic formulation of the $2 \mathrm{CH}$ system}

There are two standard ways to obtain a multi-symplectic formulation of a partial differential equation. One approach consists of using the Lagrangian formulation of the problem, see the early references $[10,20]$ and references therein. The other approach is to write the partial differential equation as a system of equations containing only first-order derivatives in space and time, see equation (2.1) below, and then to extract the multi-symplectic structure, see the early references [1,2] and references therein. In this section, we will follow the latter approach. Inspired by the multi-symplectic formulation of the Camassa-Holm equation found in [6], see also [5] for related problems, we can now derive a multi-symplectic formulation

$$
M z_{t}+K z_{x}=\nabla_{z} S(z)
$$

of the two-component Camassa-Holm system of equations

$$
\begin{aligned}
& u_{t}-u_{t x x}+3 u u_{x}=2 u_{x} u_{x x}+u u_{x x x}-\kappa \rho \rho_{x}, \\
& \rho_{t}+(u \rho)_{x}=0 .
\end{aligned}
$$

Here, $z \in \mathbb{R}^{d}$ is a vector of state variables, typically including the original variables $u$ and $\rho$ as one of its components, $M$ and $K$ are skew-symmetric $d \times d$-matrices and $S$ is a smooth scalar function depending on $z$. Indeed, setting $z=[u, \phi, w, v, \eta, \rho, \gamma, \beta]$, using the following skew-symmetric matrices

$$
M=\left[\begin{array}{cccccccc}
0 & \frac{1}{2} & 0 & 0 & -\frac{1}{2} & 0 & 0 & 0 \\
-\frac{1}{2} & 0 & 0 & 0 & 0 & 0 & 0 & 0 \\
0 & 0 & 0 & 0 & 0 & 0 & 0 & 0 \\
0 & 0 & 0 & 0 & 0 & 0 & 0 & 0 \\
\frac{1}{2} & 0 & 0 & 0 & 0 & 0 & 0 & 0 \\
0 & 0 & 0 & 0 & 0 & 0 & 0 & \frac{\kappa}{2} \\
0 & 0 & 0 & 0 & 0 & 0 & 0 & 0 \\
0 & 0 & 0 & 0 & 0 & -\frac{\kappa}{2} & 0 & 0
\end{array}\right], \quad K=\left[\begin{array}{cccccccc}
0 & 0 & 0 & -1 & 0 & 0 & 0 & 0 \\
0 & 0 & 1 & 0 & 0 & 0 & 0 & 0 \\
0 & -1 & 0 & 0 & 0 & 0 & 0 & 0 \\
1 & 0 & 0 & 0 & 0 & 0 & 0 & 0 \\
0 & 0 & 0 & 0 & 0 & 0 & 0 & 0 \\
0 & 0 & 0 & 0 & 0 & 0 & 0 & 0 \\
0 & 0 & 0 & 0 & 0 & 0 & 0 & \kappa \\
0 & 0 & 0 & 0 & 0 & 0 & -\kappa & 0
\end{array}\right]
$$

and the gradient of the scalar function

$$
\begin{gathered}
S(z)=-w u-u^{3} / 2-u \eta^{2} / 2+\eta v-\kappa u \rho^{2} / 2+\kappa \gamma \rho, \\
\text { Co-published by Atlantis Press and Taylor \& Francis } \\
\text { Copyright: the authors } \\
444
\end{gathered}
$$


one obtains a multi-symplectic formulation (2.1) of the two-component Camassa-Holm equation (1.1). The above formulation can be written componentwise:

$$
\begin{aligned}
\frac{1}{2} \phi_{t}-\frac{1}{2} \eta_{t}-v_{x} & =-w-\frac{1}{2} \eta^{2}-\frac{\kappa}{2} \rho^{2}-\frac{3}{2} u^{2}, \\
-\frac{1}{2} u_{t}+w_{x} & =0, \\
-\phi_{x} & =-u, \\
u_{x} & =\eta, \\
\frac{1}{2} u_{t} & =-u \eta+v, \\
+\frac{\kappa}{2} \beta_{t} & =\kappa \gamma-\kappa u \rho, \\
\kappa \beta_{x} & =\kappa \rho, \\
-\frac{\kappa}{2} \rho_{t}-\kappa \gamma_{x} & =0 .
\end{aligned}
$$

At this point, we would like to comment on the fact that one could obtain the above system by considering the Lagrangian

$$
\mathscr{L}:=\frac{1}{2} u \phi_{t}+\frac{1}{2} \kappa \beta_{t} \rho+\frac{1}{2} \kappa u \rho^{2}+\frac{1}{2} u^{3}+\frac{1}{2} \eta u_{t}+\frac{1}{2} u \eta^{2}+w\left(u-\phi_{x}\right)-\kappa \gamma\left(\rho-\beta_{x}\right)-v\left(\eta-u_{x}\right)
$$

and taking variations with respect to the variables $(u, \phi, w, v, \eta, \rho, \gamma, \beta)$ a A canonical way to find Lagrangians for shallow water waves can be found in [25]. In [22, Section 1.2], it is explained how to obtain a Lagrangian from a given multi-symplectic formulation.

A key observation, [2], for the above multi-symplectic formulation of our problem is that the two skew-symmetric matrices $M$ and $K$ define symplectic structures on subspaces of $\mathbb{R}^{8}$

$$
\omega=\mathrm{d} z \wedge M \mathrm{~d} z, \quad \zeta=\mathrm{d} z \wedge K \mathrm{~d} z
$$

thus resulting in the following multi-symplectic conservation law

$$
\partial_{t} \omega+\partial_{x} \zeta=0
$$

This is a local property of our problem and we thus hope that multi-symplectic numerical schemes, as derived in the next section, will render well local properties of equation (1.1). More explicitly, we have for any solutions of (2.1), the local conservation laws

$$
\partial_{t} E(z)+\partial_{x} F(z)=0 \quad \text { and } \quad \partial_{t} I(z)+\partial_{x} G(z)=0,
$$

with the density functions

$$
\begin{array}{ll}
E(z)=S(z)-\frac{1}{2} z_{x}^{T} K^{T} z, & F(z)=\frac{1}{2} z_{t}^{T} K^{T} z \\
G(z)=S(z)-\frac{1}{2} z_{t}^{T} M^{T} z, & I(z)=\frac{1}{2} z_{x}^{T} M^{T} z
\end{array}
$$

${ }^{\mathrm{a}}$ We thank the anonymous referee for providing us with this Lagrangian. 
Under the usual assumption on vanishing boundary terms for the functions $F(z)$ and $G(z)$ one obtains the following global conserved quantities

$$
\mathscr{E}(z)=\int E(z) \mathrm{d} x \quad \text { and } \quad \mathscr{I}(z)=\int I(z) \mathrm{d} x .
$$

These quantities correspond to the Hamiltonian functions (1.2) and (1.3) as we will see below. For our choice of the skew-symmetric matrices $M$ and $K$, one thus obtains the density functions

$$
\begin{aligned}
E(z)= & S(z)+\frac{1}{2} z_{x}^{T} K z=\frac{1}{4}\left(\phi_{t} u-u_{x t} u+u^{3}+u_{x} u_{t}+u u_{x}^{2}-u_{t} \phi-\kappa \rho^{2} u-\kappa \rho_{t} \beta+2 \kappa \rho \gamma\right), \\
F(z)= & -\frac{1}{2} z_{t}^{T} K z=\frac{1}{2}\left(u_{t} v-\phi_{t} w+\phi w_{t}-u v_{t}-\kappa \gamma_{t} \beta+\kappa \beta_{t} \gamma\right), \\
G(z)= & S(z)+\frac{1}{2} z_{t}^{T} M z=\frac{1}{4} \phi_{t} u-\frac{3}{4} u_{x t} u-u^{2} u_{x x}+u^{3} \\
& +\frac{1}{4} u_{x} u_{t}+\frac{1}{4} u_{t} \phi+\kappa \gamma \rho+\frac{\kappa}{4} \rho_{t} \beta-\frac{\kappa}{4} \beta_{t} \rho, \\
I(z)= & -\frac{1}{2} z_{x}^{T} M z=\frac{1}{4}\left(-u_{x} \phi+u_{x} \eta+u \phi_{x}-u \eta_{x}-\kappa \rho_{x} \beta+\kappa \rho^{2}\right) .
\end{aligned}
$$

This will help us to derive the corresponding global invariants (2.4).

We first integrate the local conservation law $\partial_{t} I(z)+\partial_{x} G(z)=0$ over the spatial domain and obtain, similarly to the computations done in [6], the invariant (1.2). Indeed, looking firstly only at terms involving $\kappa$ in the above local conservation law, one has

$$
\frac{1}{4} \frac{\mathrm{d}}{\mathrm{d} t} \int\left(-\kappa \rho_{x} \beta+\kappa \rho^{2}\right) \mathrm{d} x+\left[\kappa \gamma \rho+\frac{\kappa}{4} \rho_{t} \beta-\frac{\kappa}{4} \beta_{t} \rho\right],
$$

where the square brackets denote the difference of the function evaluated at the upper and lower limit of the integral. Using one integration by parts and the periodic boundary conditions for $u$ and $\rho$ one thus gets (removing the factor $\kappa$ for ease of presentation)

$$
\begin{aligned}
& \frac{1}{4} \frac{\mathrm{d}}{\mathrm{d} t} \int\left(\rho_{x} \beta-\rho^{2}\right) \mathrm{d} x+\left[-\gamma \rho-\frac{1}{4} \rho_{t} \beta+\frac{1}{4} \beta_{t} \rho\right] \\
& =-\frac{1}{2} \frac{\mathrm{d}}{\mathrm{d} t} \int \rho^{2} \mathrm{~d} x+\frac{1}{4} \frac{\mathrm{d}}{\mathrm{d} t}[\rho \beta]+\left[-\gamma \rho-\frac{1}{4} \rho_{t} \beta+\frac{1}{4} \beta_{t} \rho\right] \\
& =-\frac{1}{2} \frac{\mathrm{d}}{\mathrm{d} t} \int \rho^{2} \mathrm{~d} x+\frac{1}{4}\left[\rho_{t} \beta\right]+\frac{1}{4}\left[\rho \beta_{t}\right]+\left[-\gamma \rho-\frac{1}{4} \rho_{t} \beta+\frac{1}{4} \beta_{t} \rho\right] \\
& =-\frac{1}{2} \frac{\mathrm{d}}{\mathrm{d} t} \int \rho^{2} \mathrm{~d} x+\frac{1}{2}\left[\rho \beta_{t}\right]+[-\gamma \rho] \\
& =-\frac{1}{2} \frac{\mathrm{d}}{\mathrm{d} t} \int \rho^{2} \mathrm{~d} x+\left[\rho \gamma-u \rho^{2}\right]+[-\gamma \rho] \\
& =-\frac{1}{2} \frac{\mathrm{d}}{\mathrm{d} t} \int \rho^{2} \mathrm{~d} x .
\end{aligned}
$$

We next observe that

$$
\frac{1}{2} \frac{\mathrm{d}}{\mathrm{d} t} \int \kappa \rho^{2} \mathrm{~d} x
$$


is the time derivative of the third integrand present in the Hamiltonian (1.2). The two other integrands in (1.2) are obtained considering the remaining terms in the integral form of the local conservation law $\partial_{t} I(z)+\partial_{x} G(z)=0$ as this was done in [6]. This thus gives us the first Hamiltonian (1.2) of the two-component Camassa-Holm equation.

Similarly, integrating the local conservation law $\partial_{t} E(z)+\partial_{x} F(z)=0$, one obtains

$$
\begin{aligned}
0= & \frac{1}{4} \frac{\mathrm{d}}{\mathrm{d} t} \int\left(\left(\eta_{t}+2 v_{x}-2 w-\eta^{2}-\kappa \rho^{2}-3 u^{2}\right) u-u_{x t} u+u^{3}+u_{x} u_{t}+u u_{x}^{2}-2 w_{x} \phi\right. \\
& \left.-\kappa \rho^{2} u-\kappa \rho_{t} \beta+2 \kappa \rho \gamma\right) \mathrm{d} x-\frac{\kappa}{2}\left[\gamma_{t} \beta-\beta_{t} \gamma\right]+\frac{1}{2}\left[u_{t} v-\phi_{t} w+\phi w_{t}-u v_{t}\right] .
\end{aligned}
$$

Using two integrations by parts, one gets

$$
\begin{aligned}
0= & \frac{1}{4} \frac{\mathrm{d}}{\mathrm{d} t} \int\left(2 u^{2} u_{x x}+2 u u_{x}^{2}-2 u^{3}-2 \kappa \rho^{2} u+2 \kappa(\gamma \beta)_{x}\right) \mathrm{d} x \\
& +\frac{1}{4} \frac{\mathrm{d}}{\mathrm{d} t}\left[u u_{t}-2 w \phi\right]-\frac{\kappa}{2}[\ldots]+\frac{1}{2}[\ldots] \\
= & -\frac{1}{2} \frac{\mathrm{d}}{\mathrm{d} t} \int\left(u^{3}+u u_{x}^{2}+\kappa \rho^{2} u\right) \mathrm{d} x+\frac{\kappa}{2} \frac{\mathrm{d}}{\mathrm{d} t} \int(\gamma \beta)_{x} \mathrm{~d} x+\frac{1}{4} \frac{\mathrm{d}}{\mathrm{d} t}[\ldots]-\frac{\kappa}{2}[\ldots]+\frac{1}{2}[\ldots] .
\end{aligned}
$$

Finally, using the periodicity of the functions $u$ and $\rho$ (together with the periodicity of $\gamma, w, v, \phi_{t}, \beta_{t}$ ), the second integral and the expressions in the square brackets cancel and one obtains the second Hamiltonian (1.3) of the two-component Camassa-Holm equation.

\section{An Euler box scheme for the $2 \mathrm{CH}$ system}

In this section, we will derive a numerical scheme based on the multi-symplectic formulation (2.1) of the two-component Camassa-Holm equation (1.1).

Following [23], see also [19, Chap. 12], one may obtain an integrator satisfying a discrete multisymplectic conservation law by applying the classical symplectic Euler method to each independent variables in (2.1). One then obtains the so-called Euler box scheme. To do this, we first introduce finite differences. We set $\Delta x=x_{n+1}-x_{n}, n \in \mathbb{Z}$, and $\Delta t=t_{i+1}-t_{i}, i \geq 0$. Moreover, we define the forward and backward differences in time

$$
\delta_{t}^{+} Z^{n, i}=\frac{Z^{n, i+1}-Z^{n, i}}{\Delta t} \quad \text { and } \quad \delta_{t}^{-} Z^{n, i}=\frac{Z^{n, i}-Z^{n, i-1}}{\Delta t},
$$

and similarly for differences in space. The Euler box scheme uses a splitting of the two skewsymmetric matrices $M$ and $K$ in (2.1): $M=M_{+}+M_{-}$and $K=K_{+}+K_{-}$where $M_{+}^{T}=-M_{-}$and $K_{+}^{T}=-K_{-}$. This numerical scheme then reads

$$
M_{+} \delta_{t}^{+} z^{n, i}+M_{-} \delta_{t}^{-} z^{n, i}+K_{+} \delta_{x}^{+} z^{n, i}+K_{-} \delta_{x}^{-} z^{n, i}=\nabla_{z} S\left(z^{n, i}\right),
$$

where $z^{n, i} \approx z\left(x_{n}, t_{i}\right)$ on a uniform rectangular grid.

In this note, we will only consider the following matrices $M_{+}=\frac{1}{2} M$ and $K_{+}=\frac{1}{2} K$ for the above splitting, keeping in mind that the above splitting of the matrices is not unique. With this particular choice, the numerical method (3.1) now reads

$$
M \delta_{t} z^{n, i}+K \delta_{x} z^{n, i}=\nabla_{z} S\left(z^{n, i}\right)
$$

with the centered differences $\delta_{t}=\frac{1}{2}\left(\delta_{t}^{+}+\delta_{t}^{-}\right)$, and $\delta_{x}=\frac{1}{2}\left(\delta_{x}^{+}+\delta_{x}^{-}\right)$. 
For ease of implementation, we can now express the Euler box scheme (3.1) only in terms of the variables $u$ and $\rho$ and the centered divided differences $\delta_{t}$ and $\delta_{x}$ reads

$$
\begin{gathered}
\delta_{t} u^{n, i}-\delta_{t} \delta_{x}^{2} u^{n, i}+\frac{1}{2} \delta_{x}\left(\left(\delta_{x} u^{n, i}\right)^{2}\right)-\delta_{x}^{2}\left(u^{n, i} \delta_{x} u^{n, i}\right)+\frac{\kappa}{2} \delta_{x}\left(\left(\rho^{n, i}\right)^{2}\right)+\frac{3}{2} \delta_{x}\left(\left(u^{n, i}\right)^{2}\right)=0, \\
\delta_{t} \rho^{n, i}+\delta_{x}\left(u^{n, i} \rho^{n, i}\right)=0 .
\end{gathered}
$$

The multi-symplecticity of the Euler box scheme is interpreted in the sense that, recall (2.2),

$$
\delta_{t}^{+} \omega^{n, i}+\delta_{x}^{+} \zeta^{n, i}=0,
$$

where $\omega^{n, i}=\mathrm{d} z^{n, i-1} \wedge M_{+} \mathrm{d} z^{n, i}$ and $\zeta^{n, i}=\mathrm{d} z^{n-1, i} \wedge K_{+} \mathrm{d} z^{n, i}$.

Finally, we can observe that discrete versions of the above Casimir functions are preserved along the numerical solutions given by the Euler box scheme. Indeed, from equation (3.2) and using the fact that $\sum_{n} \delta_{x} v_{n}=0$ for any periodic sequence $v_{n}$, we have

$$
\delta_{t}\left(\sum_{n}\left(u^{n, i}-\delta_{x}^{2} u^{n, i}\right)\right)=0
$$

and the approximation of the Casimir function $\mathscr{C}_{2}$ given by $\sum_{n}\left(u^{n}-\delta_{x}^{2} u^{n}\right)$ is preserved in time. Similarly, we have $\delta_{t}\left(\sum_{n} \rho^{n, i}\right)=0$ and the approximation $\sum_{n} \rho^{n}$ of the Casimir function $\mathscr{C}_{1}$ is preserved in time along the numerical solutions.

\section{Numerical experiments}

\subsection{Preservation of Hamiltonians}

In the first numerical experiment that we consider, we are interested in the preservation of the invariants (1.2)-(1.3) by the numerical scheme presented in Section 3. For the initial value $u_{0}$, we take a peakon $u_{0}(x)=\exp (-|x|)$ and set $\rho_{0}=0.5$. The computational domain is set to be $[0,20]$ and the problem is integrated on the time interval $[0,5]$. Figure 1 displays snapshots of the numerical solution and the discretised Hamiltonians (corresponding to the Hamiltonians (1.2)-(1.3)),

$$
\begin{array}{r}
\mathscr{H}_{1, \Delta x}=\frac{\Delta x}{2} \sum_{n}\left(\left(u^{n}\right)^{2}+\left(\delta_{x} u^{n}\right)^{2}+\kappa\left(\rho^{n}\right)^{2}\right), \\
\mathscr{H}_{2, \Delta x}=\frac{\Delta x}{2} \sum_{n}\left(\left(u^{n}\right)^{3}+u^{n}\left(\delta_{x} u^{n}\right)^{2}+\kappa u^{n}\left(\rho^{n}\right)^{2}\right),
\end{array}
$$

along the numerical solution $\left(u^{n}, \rho^{n}\right)$ of (1.1) with $\kappa=1$ given by the Euler box scheme (3.2). Excellent conservation properties by the numerical integrator is observed. Furthermore, one can observe that the relative errors in the discrete Hamiltonians is diminishing. One interpretation could be that the numerical scheme introduces artificial numerical dissipation for this non-smooth solution. This is not the case when considering smooth solutions as the one presented at the end of this section, see the dam-break initial conditions.

The discrete versions of the Casimir functions (1.4)-(1.5) studied previously are not displayed in our plots since these quantities are exactly preserved along numerical solutions given by our multi-symplectic scheme. 

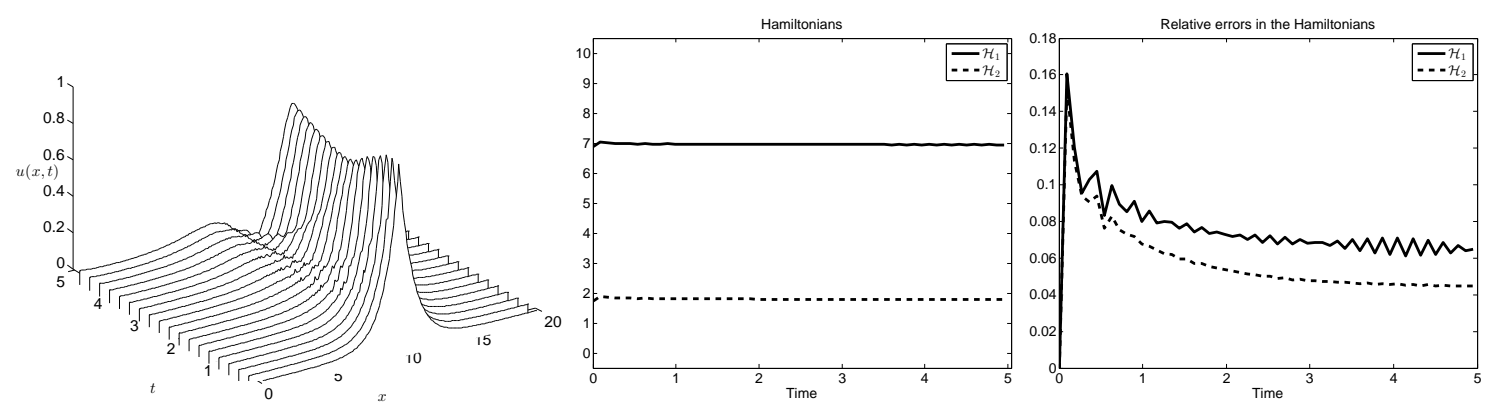

Figure 1. Peakon of 2CH: Snapshots of the numerical profile of $u$ (left plot). Discretised Hamiltonians (middle plot) and relative errors in the discrete Hamiltonians (right plot) along the numerical solution given by the Euler box scheme with $\Delta x=0.1, \Delta t=0.09$.

\subsection{Traveling wave}

Here, we present a derivation of a periodic traveling wave for (1.1) with $\kappa=1$. We look for a solution of the form $\rho(t, x)=\xi(x-c t)$ and $u(t, x)=\phi(x-c t)$. We denote $\mu=\phi-\phi^{\prime \prime}$. From (1.1), we obtain that $\xi$ and $\phi$ satisfy

$$
\begin{aligned}
\mu^{\prime}(\phi-c)+2 \phi^{\prime} \mu+\kappa \xi \xi^{\prime} & =0 \\
-c \xi^{\prime}+(\phi \xi)^{\prime} & =0
\end{aligned}
$$

We introduce $\tilde{\phi}=\phi-c$ and, correspondingly, $\tilde{\mu}=\mu-c$. The system above, after multiplying the first equation with $\tilde{\phi}$, can the be rewritten as

$$
\begin{aligned}
\left(\tilde{\phi}^{2}(\tilde{\mu}+c)\right)^{\prime}+\kappa \tilde{\phi} \xi \xi^{\prime} & =0, \\
(\tilde{\phi} \xi)^{\prime} & =0 .
\end{aligned}
$$

We integrate the second equation and obtain

$$
\tilde{\phi} \xi=A
$$

for some real constant of integration $A$. We plug this result into the first equation and get

$$
\left(\tilde{\phi}^{2}(\tilde{\mu}+c)\right)^{\prime}+(\kappa A \xi)^{\prime}=0
$$

which, after integration, yields

$$
\tilde{\phi}^{2} \tilde{\mu}+c \tilde{\phi}^{2}+\kappa A \xi=B
$$

for a constant of integration $B$. Using (4.1) and the definition of $\mu$, we finally obtain the following second order differential equation for $\phi$

$$
\phi^{\prime \prime}=\kappa \frac{A^{2}}{(\phi-c)^{3}}-\frac{B}{(\phi-c)^{2}}+\phi .
$$

Let $f(\phi)=\kappa \frac{A^{2}}{(\phi-c)^{3}}-\frac{B}{(\phi-c)^{2}}+\phi$. Only periodic solutions to the above differential equation will give us traveling waves for $2 \mathrm{CH}$. For $\kappa=1$ and $c=-A=-B=2$, we numerically check that the solution of (4.2) for $\phi(0)=0.5$ and $\phi^{\prime}(0)=0$ is periodic with period 5.1475. Using (4.1), we obtain 

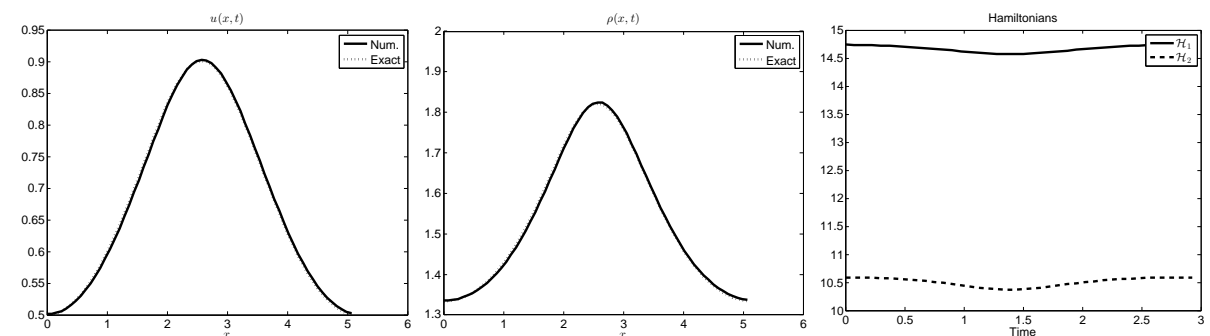

Figure 2. Traveling wave of 2CH: Exact and numerical profiles of $u(x, t)$ and $\rho(x, t)$ at time $T=3$ and discrete Hamiltonians (right plot).

$\xi=\frac{A}{\phi-c}$. We use this solution as reference solution in our code. A similar derivation of traveling waves for the two-component Camassa-Holm equation was obtained in [7].

Figure 2 displays the exact and numerical profiles of $u(x, t)$ and $\rho(x, t)$ at time $T=3$ and also the computed discrete versions of the Hamiltonians (1.2)-(1.3) using the Euler box scheme (3.2) with step sizes $\Delta t=0.06$ and $\Delta x=0.09$. One may notice that the numerical solution agrees very well with the exact ones and also good conservation properties of the numerical scheme.

\subsection{Peakon anti-peakon solution}

Since the Camassa-Holm equation is obtained from (1.1) setting $\rho \equiv 0$, it is interesting to consider how peakon anti-peakon solution initial value behaves for the system (1.1) with $\kappa=1$. In Figure 3, we thus consider the following initial value (see also [12])

$$
\begin{aligned}
& u_{0}= \begin{cases}1 / \sinh (1 / 4) \sinh (x) & \text { if } x \geq 0 \text { and } x \leq 1 / 4 \\
\sinh (x-1 / 2) / \sinh (-1 / 2) & \text { if } x>1 / 4 \text { and } x \leq 3 / 4 \\
1 / \sinh (1 / 4) \sinh (x-1) & \text { if } x>3 / 4 \text { and } x<1\end{cases} \\
& \rho_{0}=1.5
\end{aligned}
$$

and displays the numerical solution obtained by our multi-symplectic scheme with meshes $\Delta t=$ 0.003 and $\Delta x=0.004$ on the periodic domain $[0,1]$. Since $\rho_{0}>0$, it can be shown that $\rho(t)$ remains strictly positive and that the solution retains the regularity of the initial data, see [11]. In particular $\rho(t)$ remains bounded. As expected, we observe that the numerical approximation of $\rho$ concentrates around the collision point of the peakon anti-peakon case of the $\mathrm{CH}$ equation (case $\rho=0$ ). It corresponds to the fact that, at that time, the total energy distribution $u^{2}+u_{x}^{2}+\rho^{2}$ becomes mainly supported by the variable $\rho$. Despite this concentration phenomena, we still observe good preservation properties for the numerical scheme.

\subsection{Dam-break initial conditions}

Finally, we consider problem (1.1) with $\kappa=1$ on the periodic domain $[-6,6]$ augmented with the dam-break initial conditions from [15]

$$
u(x, 0)=0, \quad \rho(x, 0)=1+\tanh (x+0.1)-\tanh (x-0.1) .
$$

Figure 4 displays the evolution of $u(x, t)$ and $\rho(x, t)$ on the time interval $[0,20]$ together with the computed discrete versions of the Hamiltonians (1.2)-(1.3) for the numerical solution given by the 

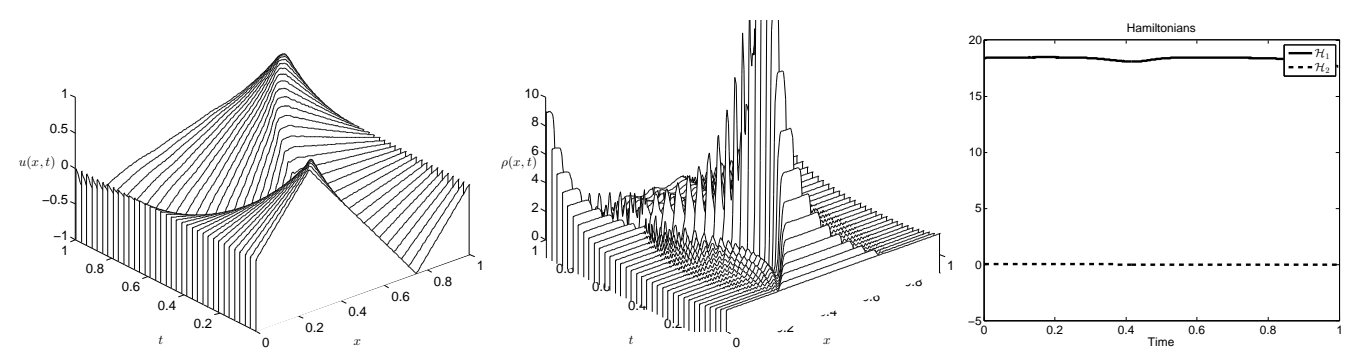

Figure 3. Peakon anti-peakon of $2 \mathrm{CH}$ : Evolution of the numerical profiles of $u(x, t)$ and $\rho(x, t)$ on the time interval $[0,1]$ and discrete Hamiltonians (right plot).
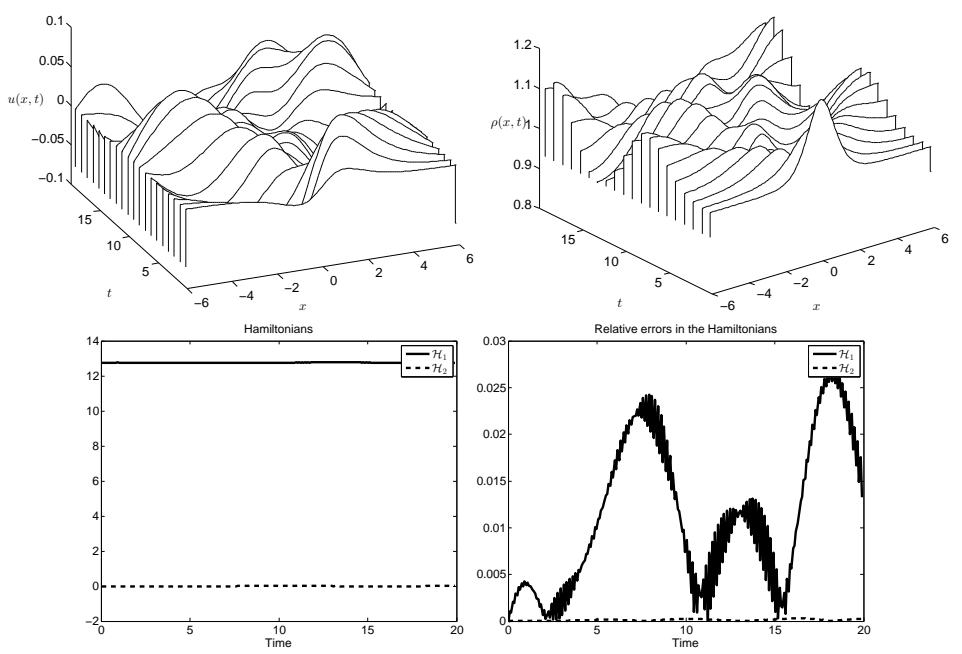

Figure 4. Dam-break of 2CH: Evolution of the numerical profiles of $u(x, t)$ and $\rho(x, t)$ on the time interval [0,20] (top). Discrete Hamiltonians and relative errors in the discrete Hamiltonians (bottom).

Euler box scheme using step sizes $\Delta t=0.08$ and $\Delta x=0.09$. Once again, the numerical solution conserves very well the discrete Hamiltonians of the $2 \mathrm{CH}$ system. Furthermore, in contrast with the first numerical experiment on the peakon solution, the relative errors in this case do not diminish since we consider smooth solution.

\section{Conclusion and open problems}

With this note, we have presented the first multi-symplectic formulation of the two-component Camassa-Holm equation. This motivates the use of multi-symplectic integrators for the numerical discretisation of this system of partial differential equations. Furthermore, good conservation properties in terms of energies were illustrated by the (multi-symplectic) Euler box scheme. Finally, it was shown that the above numerical method exactly preserves two discrete versions of the Casimir functions of the problem. 
Using the framework of discrete variational derivative methods [9], one could in principle construct $\mathscr{H}_{1, \Delta x}$ or $\mathscr{H}_{2, \Delta x}$-preserving numerical schemes for $2 \mathrm{CH}$. Such numerical schemes will however not be multi-symplectic in general. It would be of interest to get more insight into the (longtime) behaviour of such energy preserving schemes in comparison to the multi-symplectic Euler box scheme presented here. Numerical comparisons of both types of methods is proposed in [5] for Hunter-Saxton like equations.

Furthermore, one could extend the multi-symplectic Euler box scheme derived in the present article to numerically integrate the generalised two-component Camassa-Holm equation [4]

$$
\begin{aligned}
& u_{t}-u_{t x x}+3 u u_{x}-A u_{x}=2 u_{x} u_{x x}+u u_{x x x}-\kappa \rho \rho_{x}, \\
& \rho_{t}+(u \rho)_{x}=0,
\end{aligned}
$$

where $A \geq 0$ without additional difficulties. It is however not clear to the authors if one can get a second multi-symplectic formulation of our problem based on the reformulation of the $2 \mathrm{CH}$ system [11]

$$
\begin{aligned}
& u_{t}+u u_{x}+P_{x}=0, \\
& \rho_{t}+(u \rho)_{x}=0, \\
& P-P_{x x}=u^{2}+\frac{1}{2} u_{x}^{2}+\kappa \frac{1}{2} \rho^{2}, \\
& \mu_{t}+(u \mu)_{x}=\left(u^{3}-2 P u\right)_{x},
\end{aligned}
$$

with the measure $\mu:=u^{2}+u_{x}^{2}+\kappa \rho^{2}$. Such formulation would allow to compute the conservative solutions of the $2 \mathrm{CH}$ equation, as it was done in [6] for the $\mathrm{CH}$ equation. Conservative solutions are global, weak, energy preserving solutions, defined beyond the blows up which may naturally arise for this equation, see $[12,13]$.

\section{Acknowledgement}

We greatly appreciate the referees' comments on an earlier version. DC acknowledges support from UMIT Research Lab at Umeå University and the FY2012 Researcher Exchange Program between the Japan Society for the Promotion of Science and the Royal Swedish Academy of Sciences.

\section{References}

[1] T. J. Bridges, Multi-symplectic structures and wave propagation, Math. Proc. Cambridge Philos. Soc. 121(1) (1997) 147-190.

[2] T. J. Bridges and S. Reich, Multi-symplectic integrators: numerical schemes for Hamiltonian PDEs that conserve symplecticity, Phys. Lett. A 284(4-5) (2001) 184-193.

[3] M. Chen, S.-Q. Liu and Y. Zhang, A two-component generalization of the Camassa-Holm equation and its solutions, Lett. Math. Phys. 75(1) (2006) 1-15.

[4] R. M. Chen and Y. Liu, Wave breaking and global existence for a generalized two-component CamassaHolm system, Int. Math. Res. Not. IMRN (6) (2011) 1381-1416.

[5] D. Cohen, D. Furihata, T. Matsuo and Y. Miyatake, Numerical integrators for Hunter-Saxton-like equations, submitted for publication (2013).

[6] D. Cohen, B. Owren and X. Raynaud, Multi-symplectic integration of the Camassa-Holm equation, $J$. Comput. Phys. 227(11) (2008) 5492-5512.

[7] A. Constantin and R. I. Ivanov, On an integrable two-component Camassa-Holm shallow water system, Phys. Lett. A 372(48) (2008) 7129-7132. 
[8] J. Escher, M. Kohlmann and J. Lenells, The geometry of the two-component Camassa-Holm and Degasperis-Procesi equations, J. Geom. Phys. 61(2) (2011) 436-452.

[9] D. Furihata and T. Matsuo, Discrete Variational Derivative Method (CRC Press, Boca Raton, FL, 2011). A structure-preserving numerical method for partial differential equations.

[10] M. J. Gotay, A multisymplectic framework for classical field theory and the calculus of variations. I. Covariant Hamiltonian formalism, in Mechanics, analysis and geometry: 200 years after Lagrange, North-Holland Delta Ser. (North-Holland, Amsterdam, 1991) pp. 203-235.

[11] K. Grunert, H. Holden and X. Raynaud, Global solutions for the two-component Camassa-Holm system, Comm. Partial Differential Equations 37(12) (2012) 2245-2271.

[12] K. Grunert, H. Holden and X. Raynaud, Periodic conservative solutions for the two-component Camassa-Holm system, in Spectral Analysis, Differential Equations and Mathematical Physics, Proc. Symp. Pure Math. 87 (Amer. Math. Soc., 2013) pp. 165-182.

[13] G. Gui and Y. Liu, On the global existence and wave-breaking criteria for the two-component CamassaHolm system, J. Funct. Anal. 258(12) (2010) 4251-4278.

[14] Z. Guo, Asymptotic profiles of solutions to the two-component Camassa-Holm system, Nonlinear Anal. 75(1) (2012) 1-6.

[15] D. D. Holm and R. I. Ivanov, Multi-component generalizations of the CH equation: geometrical aspects, peakons and numerical examples, J. Phys. A 43(49) (2010) 492001, 20.

[16] D. D. Holm and R. I. Ivanov, Smooth and peaked solitons of the CH equation, J. Phys. A 43(43) (2010) 434003, 18.

[17] D. D. Holm and R. I. Ivanov, Two-component CH system: inverse scattering, peakons and geometry, Inverse Problems 27(4) (2011) 045013, 19.

[18] R. Ivanov, Two-component integrable systems modelling shallow water waves: the constant vorticity case, Wave Motion 46(6) (2009) 389-396.

[19] B. Leimkuhler and S. Reich, Simulating Hamiltonian Dynamics (Cambridge University Press, 2004).

[20] J. E. Marsden, G. W. Patrick and S. Shkoller, Multisymplectic geometry, variational integrators, and nonlinear PDEs, Comm. Math. Phys. 199(2) (1998) 351-395.

[21] K. Mohajer, A note on traveling wave solutions to the two component Camassa-Holm equation, $J$. Nonlinear Math. Phys. 16(2) (2009) 117-125.

[22] B. E. Moore, A Modified Equations Approach for Multi-Symplectic Integration Methods, PhD thesis, University of Surrey, (Surrey, UK, 2003).

[23] B. E. Moore and S. Reich, Backward error analysis for multi-symplectic integration methods, Numer. Math. 95(4) (2003) 625-652.

[24] O. G. Mustafa, On smooth traveling waves of an integrable two-component Camassa-Holm shallow water system, Wave Motion 46(6) (2009) 397-402.

[25] Y. Nutku, Canonical formulation of shallow water waves, J. Phys. A 16(18) (1983) 4195-4201.

[26] P. J. Olver and P. Rosenau, Tri-hamiltonian duality between solitons and solitary-wave solutions having compact support, Phys. Rev. E 53(Feb 1996) 1900-1906.

[27] X. Raynaud, On a Shallow Water Wave Equation, PhD thesis, NTNU Trondheim, (Trondheim, NO, 2006). 